Buxton, E. W. (1960). J. gen. Microbiol. 22, 678-689

\title{
Effects of Pea Root Exudate on the Antagonism of Some Rhizosphere Micro-organisms towards Fusarium oxysporum f. pisi
}

\author{
By E. W. BUXTON \\ Rothamsted Experimental Station, Harpenden, Hertfordshire
}

SUMMARY: The microbial populations of rhizosphere soil from pea varieties Onward, susceptible to wilt by Fusarium oxysporum f. pisi race 1, and Wilt-Resistant Alaska were assessed at six successive plant-growth stages, by the dilution plate technique. The most commonly found rhizosphere organisms were: Gliocladium roseum, Penicillium spp., bacteria, Fusarium roseum, $F$. oxysporum, $F$. solani, Mortierella spp., Rhizopus stolonifer and Trichoderma viride. Root exudate from variety Onward stimulated growth and sporulation of some of the more prevalent 24 of 60 different species among 137 morphologically different isolates of fungi and bacteria. Testing the rhizosphere isolates for their in vitro effects on the pathogenic Fusarium showed that $15 \%$ had no effect, $29 \%$ were slightly inhibitory, $42 \%$ considerably more and $14 \%$ strongly inhibitory. Prominent among the strongest inhibitors were a few bacteria, Gliocladium roseum and strains of $F$. oxysporum, the last showing that intraspecific inhibition occurs among the Fusaria.

When ten of the more prevalent rhizosphere fungi were grown in media containing rhizosphere soil extract, root exudate or both, their ability to inhibit the pathogenic Fusarium greatly increased; the most inhibition was obtained with culture filtrates of fungi grown in the presence of both rhizosphere soil extract and root exudate. Morphologically different isolates of Gliocladium roseum and Fusarium oxysporum inhibited the pathogenic Fusarium to different extents, showing that these species contain physiologic strains that could act differentially towards $F$. oxysporum f. pisi in the rhizosphere.

The rhizosphere of the wilt-resistant variety Alaska contained no more inhibitory isolates than the rhizosphere of the wilt-susceptible Onward. Although there were more micro-organisms per unit of dry rhizosphere soil of the susceptible variety, the species isolated from the rhizospheres of the susceptible and resistant varieties did not differ qualitatively.

Rhizosphere micro-organisms that were more prevalent up to the time that Fusarium invaded the host roots were not prominent among the group most antagonistic towards the Fusarium. In addition, there was no correlation between stimulation of rhizosphere organisms by root exudate and their antagonism towards the Fusarium. This implies that competition between pathogenic Fusarium and the other rhizosphere micro-flora for nutrients in root exudates may be at least as important as overcoming antibiosis in maintaining successful growth of the pathogen near hostroot surfaces. Although these results were obtained in vitro, they suggest that rhizosphere soil extract and microbial metabolites together deter the growth of Fusarium oxysporum f. pisi near pea roots, and that the growth-promoting effects of root exudate from the wilt-susceptible pea variety Onward are partially offset by its ability to increase inhibition by some of the other rhizosphere inhabitants.

The rhizosphere constitutes the zone of intense microbial activity near root surfaces and exists primarily because living roots exude materials into the soil. The root exudates, together with breakdown products from cells detached from root surfaces during growth, stimulate the rhizosphere microflora; 
they affect both pathogenic and non-pathogenic micro-organisms that occur near roots of potential hosts (Timonin, 1941; Rovira, 1956; Buxton, 1957 a). A study of the factors which affect any root pathogen in the rhizosphere must include these non-pathogens, because it is in the rhizosphere that the general microflora will most affect the pathogen's ability to survive and grow and so determine its capacity to begin the cycle of host infection.

The status of Fusarium oxysporum $\mathrm{f}$. pisi race 1 (the cause of pea wilt) is considered here in relation to some of the biochemical factors that may affect its growth and activity in the rhizosphere of pea (Pisum sativum L.). The direct effects of pea-root exudates on this fungus may be mitigated by the metabolic products of prevalent rhizosphere micro-organisms. These, in turn, may have additional effects on the Fusarium, depending on whether or not root exudates may increase their growth rates or their output of antibiotic metabolites. A broad outline of the postulated interactions between these factors in the rhizosphere and their possible effects on a pathogenic Fusarium can be expressed diagrammatically:

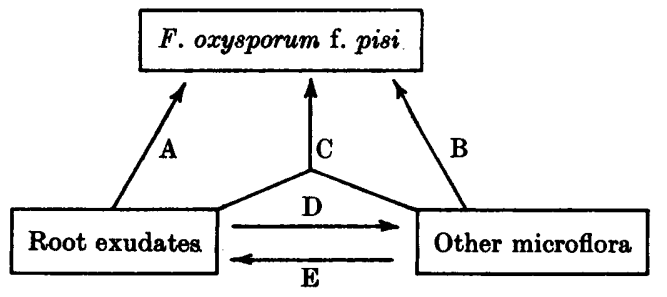

Root exudates can directly affect both the pathogenic Fusarium and the other rhizosphere microflora (A and D). The microflora will affect the Fusarium (B) and, when supplied with nutrients contained in root exudate, they may have additional effects (C). Interactions $A, B$, and $D$ are primary, whereas $C$ may be additional to $B$, depending on the availability and type of nutrients in the root exudate. Whether or not effect $\mathbf{E}$ can be assessed, that is whether any of the rhizosphere microflora may alter the quantity or quality of root exudate, depends on the evolution of a suitable technique. The evidence that the soil microflora may be antagonistic to or may compete against root pathogens in the rhizosphere was reviewed by Garrett (1956), but the effects root exudates may have on microbial antibiosis seem not to have been studied.

\section{METHODS}

Rhizosphere microflora. Samples of pea rhizosphere soils, consisting of the particles of soil adhering to roots after shaking off all surplus soil, were taken on six occasions during the growth of pea varieties Onward (wilt-susceptible) and Wilt-Resistant Alaska, grown in soil obtained from a field naturally infected with Fusarium oxysporum f. pisi race 1, at Yaxley, Peterborough. Using the methods previously described (Buxton, 1957b), soil dilutions of 1/10,000 were spread in Petri dishes containing $15 \mathrm{ml}$. of Czapek-Dox + yeastextract agar medium as used by Warcup (1950). Preliminary experiments 
showed that the dilution used allowed growth of 20 to 30 colonies of soilborne organisms on each dish. After incubation for 4 days at $25^{\circ}$, the cultures were examined and recorded, and after a further 5 days in daylight at room temperature, they were re-examined for slow-growing colonies and the development of any other taxonomic characters. Subcultures were made to slopes of Czapek-Dox agar medium to confirm identifications and for subsequent experimental work. Each sample at this stage of the work involved the use of 60 Petri dishes; the experiments were repeated on two other occasions.

Interactions between rhizosphere microflora and Fusarium oxysporum $f$. pisi. Antibiotic effects of the rhizosphere microflora on the pathogenic Fusarium were tested for by two methods: (1) by counting Fusarium spore germination in cell-free culture extracts of each rhizosphere isolate; (2) by assessing the effects of culture filtrates from each isolate on the growth of the Fusarium in agar media. Preliminary experiments showed that the agar-plate test was both more rapid and gave more consistent results than tests involving laborious counts of germinating spores. This being so, and although similar results were obtained by each method, the agar plate tests were repeated and results from them alone are reported here.

The culture filtrates of the various rhizosphere isolates were prepared by growing them in each of the media shown in Table 1.

Table 1. Culture media used for testing the interactions of rhizosphere organisms and Fusarium oxysporum $f$. pisi race 1

$\begin{array}{cl}\begin{array}{c}\text { No. of } \\ \text { method }\end{array} & \begin{array}{c}\text { Liquid medium used for } \\ \text { culturing organism } \\ \text { Czapek-Dox } \\ \text { Czapek-Dox } \\ \mathbf{2}\end{array} \\ \mathbf{3} & \begin{array}{l}\text { Rhizosphere soil extract } \\ \text { Rhizosphere soil extract } \\ \text { +root exudate }\end{array}\end{array}$

Root exudates were prepared in a soil-perfusion apparatus (Pl. 1, fig. 2). Culture filtrates and root exudates were both concentrated in vacuo at $40^{\circ}$ and passed through Oxoid bacteriological membrane filters, to obtain cellfree extracts and to ensure their sterility. In the agar-plate tests for inhibition, the test fluids were put into holes $7 \mathrm{~mm}$. diameter and $5 \mathrm{~mm}$. deep, punched in the agar medium, into which spores of Fusarium had been dispersed at pouring. Typical effects of root exudates from the two pea varieties on the growth of the two physiologic races of the Fusarium are shown in Pl. 1, fig. 1.

\section{RESULTS}

\section{Isolates from rhizosphere soils}

A total of 137 fungi of different appearance, together with a few bacteria, were isolated from rhizosphere soils. The most prevalent fungi were identified (Table 2), and the bacteria and a few actinomycetes were retained for testing at a later stage. No attempt was made to identify fungi which occurred only 
rarely. The frequency with which these fungi occurred in the rhizosphere of varieties Onward and Wilt-Resistant Alaska at different sample times is shown in Fig. 1.

Table 2. Prevalent species of organisms isolated from the rhizosphere soil of pea variety Onward growing in wilt-infested soil from Yaxley, Peterborough

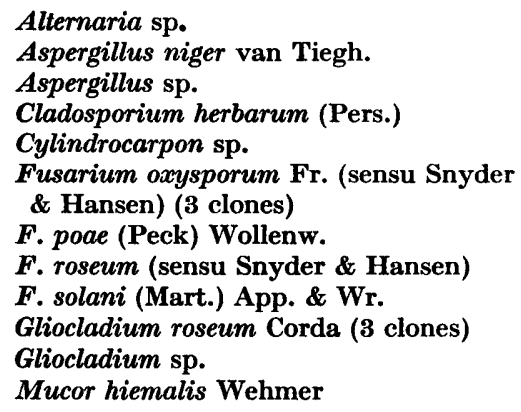

\author{
Mortierella spp. \\ Oospora crustacea \\ Penicillium lilacinum Thom \\ P. luteum Zukal \\ Penicillium spp. \\ Phoma spp. \\ Rhizoctonia solani Kühn \\ Rhizopus stolonifer (Fr.) Lind. \\ Stachybotrys atra Corda \\ Trichoderma lignorum (Tode) Harz. \\ T. viride Pers. ex Fr. \\ Verticillium sp. \\ Various actinomycetes and bacteria
}

The effect of root exudate on the prevalent species of the rhizosphere microflora

Using the agar-plate test for the effects of root exudates from pea variety Onward on its rhizosphere micro-organisms the reactions of 24 different isolates of the most prevalent of more than 60 different species were examined. Both the width of the zones of reaction and the intensity of inhibition or stimulation were recorded (Table 3). Fifteen of the 24 isolates were stimulated by root exudate, nine showed no response and none was inhibited. The stimulation of growth (Pl. 1, fig. 3), accompanied in some isolates by increased sporulation, clearly affords these rhizosphere organisms a distinct ecological advantage. Experiments were made to see whether such advantages were expressed at the expense of the pathogenic Fusarium.

Tests for activity of rhizosphere organisms against $\mathbf{F}$. oxysporum $f$. pisi

All the rhizosphere isolates were tested in vitro to see whether they showed antibiosis against Fusarium oxysporum f. pisi. This was done by streaking each isolate along a diameter of a Petri dish containing Czapek-Dox agar medium, parallel to two streaks of the Fusarium, each $2 \mathrm{~cm}$. away on either side. The results were assessed as shown in Table 4.

Fourteen percent of the rhizosphere organisms inhibited growth of the Fusarium strongly (grades 4 and 5), and $60 \%$ to the extent of grades 2 and 3. One surprising and interesting feature of this test was that 22 out of 24 of the soil isolates of Fusarium oxysporum inhibited the pathogenic strain of $F$. oxysporum f. pisi. Only three of the bacteria were among the most inhibitory organisms, and 16 out of 20 isolates of Gliocladium were fairly inhibitory. But in tests of this kind it must be emphasized that the inhibitory effects observed in vitro are not necessarily the same as any that may occur in the rhizosphere; conversely effects in soil may not be detectable by agar-plate techniques. 
Table 3. Effects of root exudate from pea variety Onward on prevalent pea rhizosphere organisms, assessed by zone of reaction on Czapek-Dox agar medium

\begin{tabular}{l} 
Rhizosphere organism \\
\multicolumn{1}{c}{ Fusarium oxysporum no. 1 (pink) } \\
F. oxysporum no. 2 (white) \\
F. oxysporum no. 3 \\
F. oxysporum no. 4 \\
Fusarium poae \\
Penicillium sp. no. 1 \\
Penicillium sp. no. 2 \\
P. lilacinum \\
Verticillium sp. \\
'Black fungus' (unidentified) \\
Cliocladium roseum no. 1 \\
G. roseum no. 2 \\
G. roseum no. 3 \\
Gliocladium sp. \\
Phoma sp. \\
Trichoderma viride \\
Stachybotrys atra \\
Unknown: \\
BB4 \\
U4 \\
U5 \\
U36 \\
U40/2 \\
U44 \\
U45
\end{tabular}

\begin{tabular}{|c|c|}
\hline \multicolumn{2}{|c|}{ Stimulation } \\
\hline $\begin{array}{c}\text { Intensity } \\
(0-5)\end{array}$ & $\begin{array}{c}\text { Width } \\
\text { of zone } \\
\text { (mm.) }\end{array}$ \\
\hline 4 & 27 \\
\hline 2 & 22 \\
\hline 2 & 11 \\
\hline 5 & 24 \\
\hline 1 & 15 \\
\hline 5 & 13 \\
\hline 0 & 0 \\
\hline 0 & 0 \\
\hline 1 & 24 \\
\hline 5 & 12 \\
\hline 3 & 20 \\
\hline 0 & 0 \\
\hline 4 & 22 \\
\hline 0 & 0 \\
\hline 3 & 13 \\
\hline 1 & 9 \\
\hline 4 & 21 \\
\hline 0 & 0 \\
\hline 0 & o \\
\hline 0 & 0 \\
\hline 0 & 0 \\
\hline 1 & 9 \\
\hline 4 & 15 \\
\hline 0 & 0 \\
\hline
\end{tabular}
Additional effects
Increased aerial mycelium
Tendency to grey colour
Slightly increased sporulation
White zonation
Slight extra growth
-
Very slight extra growth
Increased sporulation
Increased depth of colour
Very slight extra growth
Increased depth of colour

\section{Comparison of rhizosphere microflora from pea varieties Onward and Wilt-Resistant Alaska}

Differences in the microflora that inhabit the rhizospheres of different hosts might affect the degree of host resistance to soil-borne pathogens, so it was of interest to see whether the rhizosphere microflora of a wilt-susceptible and wilt-resistant pea differed either in the prevalence of different species or in the abilities of the different components to inhibit growth of the pathogenic Fusarium. The predominant organisms able to inhibit the pathogenic Fusarium (Table 4) were selected and compared; differences in frequency between some of the more frequent groups in the rhizosphere of the two varieties Onward and Wilt-Resistant Alaska are shown in Fig. 1.

As Fusarium oxysporum is prevalent in the soil used, it might be expected to provide a large proportion of the total rhizosphere organisms. The occurrence of Gliocladium, Trichoderma and Penicillium spp. and the increase of $F$. oxysporum at the beginning of the growth of the host have previously been recorded in the pea-root microflora (Buxton, 1957b). Stenton (1958) found that Cladosporium and species of Fusarium were the two most prevalent fungi on pea-root surfaces during the 3rd to 7th weeks of growth, but the frequency with which he recorded Cylindrocarpon contrasts with the results recorded 
Table 4. Results of agar-plate tests of groups of rhizosphere organisms for antibiotic effects on Fusarium oxysporum $f$. pisi race 1

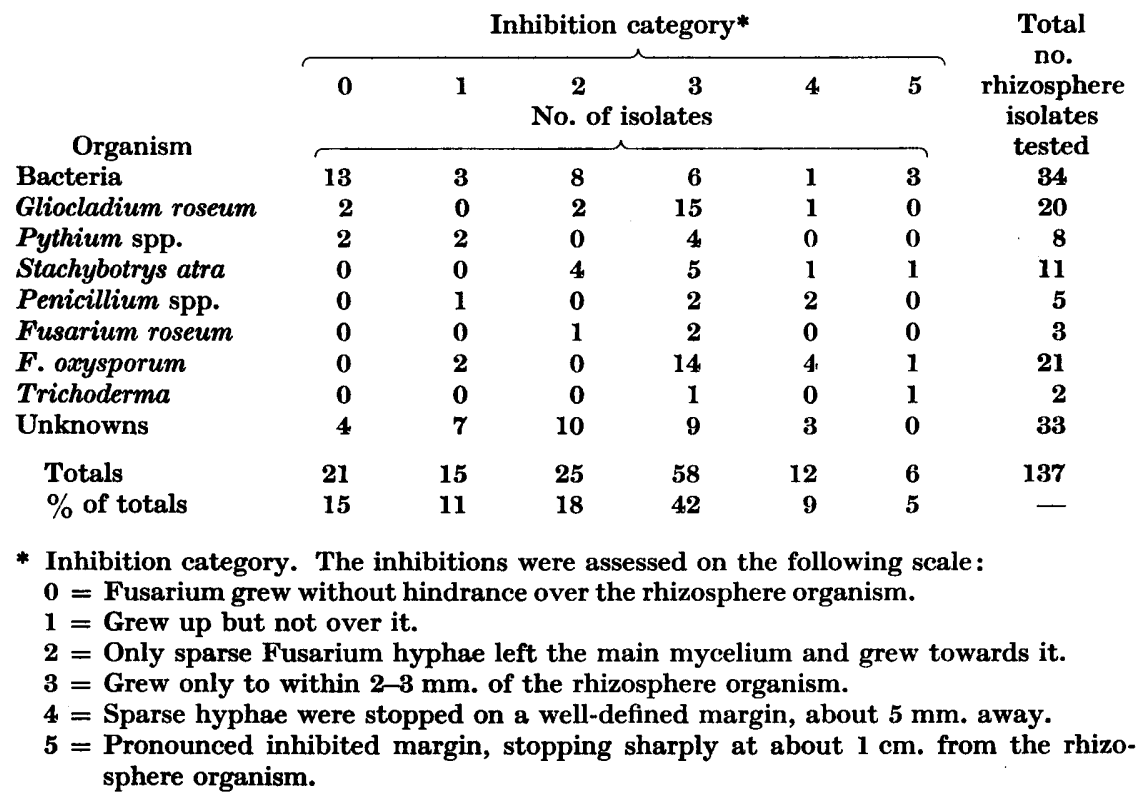

here for rhizosphere soil and with earlier records of pea-root surface fungi. Nevertheless, few comparisons, and even fewer valid conclusions, can be made from the existing agar-plating techniques for assessing the prevalence of root surface or rhizosphere fungi, because too much depends on the selective action of the media, the conditions of plant growth, and the numbers of replicates used by each experimenter. The only consistent differences of any magnitude between the frequencies of the rhizosphere microflora of the two varieties showed in Gliocladium and Penicillium and in the total of all the organisms, which is greater from the wilt-susceptible variety Onward than from WiltResistant Alaska. Both Gliocladium and Penicillium contained species and strains which inhibited the pathogenic Fusarium (Table 4), but a preponderance of one and a decrease in the other might cancel out any possible disadvantage to the Fusarium that may occur in the rhizosphere of the resistant variety.

Inhibitory strains of Trichoderma and Stachybotrys are more frequent in the rhizosphere of the resistant variety than in that of the susceptible variety towards the end of the life of the plant. But they are unlikely to add to the effect of any resistance factors in the root exudate because their inhibitory effects in the rhizosphere can obviously be effective only when they occur relatively early in the life of the host plant, before the Fusarium invades the root. Chromatographic analyses of root exudates from the two pea varieties used showed that they contain similar amino acids and sugars, so a pronounced 

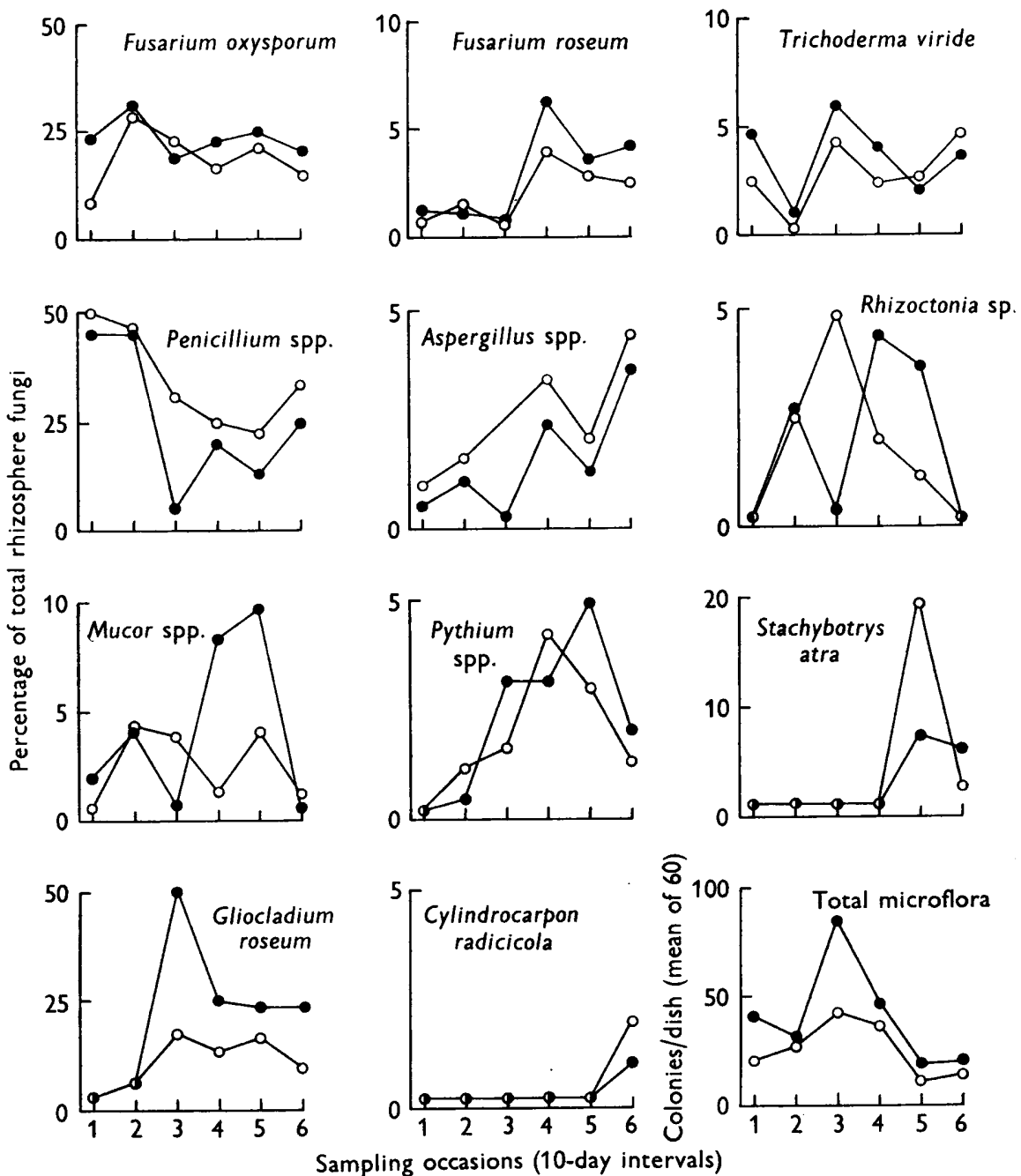

Fig. 1. Percentage frequencies of total organisms isolated from rhizosphere of pea varieties Onward (Fusarium race 1-susceptible) and Wilt-Resistant Alaska (Fusarium race 1resistant) on six successive occasions 10 days apart. Note changes in scale of ordinates. 0 variety Onward; $O=$ variety Wilt-Resistant Alaska.

qualitative difference in their rhizosphere microflora, correlated with differences in their gross nutritional requirements, could not be expected.

There is, then, no evidence that the rhizosphere of the wilt-resistant pea variety preferentially encourages those components of the rhizosphere microflora which inhibit the Fusarium. This suggests that in a pea variety, resistance to infection, at least at root level, operates directly on the pathogen and is not primarily affected by the various components of the rhizosphere microflora which inhibit Fusarium oxysporum f. pisi. Rombouts (1953) similarly found 
that rhizosphere microflora which inhibited $F$. oxsyporum f. cubense (the cause of banana wilt) occurred with equal frequency near roots of both wilt-susceptible and wilt-resistant banana.

Effects of root exudate and soil extract on inhibition of $\mathbf{F}$. oxysporum $f$. pisi by rhizosphere organisms

To test for any modifying effects that Onward root exudate and rhizosphere soil extracts may have on the inhibition of Fusarium oxysporum f. pisi race 1 by culture filtrates of rhizosphere fungi, the various mixtures of root exudate, soil extract and microbial metabolites that may affect the pathogenic Fusarium near roots were used as culture media and test fluids (Table 1). Changes in the inhibitory effects of ten of the most prevalent rhizosphere organisms were assessed (Table 5).

Table 5. Effects of pea-root exudates and pea rhizosphere soil extracts on the inhibition of Fusarium oxysporum $f$. pisi by culture filtrates of pea rhizosphere micro-organisms

\begin{tabular}{|c|c|c|c|c|c|c|c|c|}
\hline & \multicolumn{8}{|c|}{ Test method used (see Table 1) } \\
\hline & \multicolumn{2}{|c|}{1} & \multicolumn{2}{|c|}{$\mathbf{2}$} & \multicolumn{2}{|c|}{$\mathbf{3}$} & \multicolumn{2}{|c|}{4} \\
\hline & I & $\mathbf{s}$ & $\mathbf{I}$ & $\mathbf{S}$ & I & $\mathbf{s}$ & I & $\mathbf{s}$ \\
\hline Verticillium sp. & $\mathbf{0}$ & $\mathbf{0}$ & $\begin{array}{c}1 \\
(8 \cdot 0)\end{array}$ & $\mathbf{0}$ & $\mathbf{0}$ & $\mathbf{0}$ & $\begin{array}{c}5 \\
(18 \cdot 5)\end{array}$ & $\mathbf{0}$ \\
\hline U 40/2 (unidentified) & $\mathbf{0}$ & $\mathbf{0}$ & $\begin{array}{c}1 \\
(12 \cdot 0)\end{array}$ & $\mathbf{0}$ & $\begin{array}{c}3 \\
(\mathbf{1 3} \cdot \mathbf{0})\end{array}$ & $\mathbf{0}$ & $\begin{array}{c}4 \\
(12 \cdot 0)\end{array}$ & $\mathbf{0}$ \\
\hline Gliocladium roseum 1 & $\begin{array}{c}1 \\
(8 \cdot 5)\end{array}$ & $\mathbf{0}$ & $\mathbf{0}$ & $\mathbf{0}$ & $\begin{array}{c}2 \\
(9 \cdot 0)\end{array}$ & $\mathbf{0}$ & $\stackrel{4}{(15 \cdot 0)}$ & $\mathbf{0}$ \\
\hline G. roseum 2 & $\begin{array}{c}1 \\
(9 \cdot 0)\end{array}$ & $\begin{array}{c}0 \\
(\mathbf{1 2 \cdot 0})\end{array}$ & 1 & $\mathbf{0}$ & $\mathbf{0}$ & $\begin{array}{c}3 \\
(\mathbf{1 2} \cdot \mathbf{0})\end{array}$ & $\stackrel{4}{4}$ & $\mathbf{0}$ \\
\hline G. roseum 3 & $\begin{array}{c}3 \\
\mathbf{( 1 4 \cdot 0 )}\end{array}$ & $\mathbf{0}$ & $\mathbf{0}$ & $\mathbf{0}$ & $\begin{array}{c}5 \\
(13 \cdot 0)\end{array}$ & $\mathbf{0}$ & $\begin{array}{c}4 \\
(\mathbf{1 3} \cdot 0)\end{array}$ & $\mathbf{0}$ \\
\hline Gliocladium sp. & $\begin{array}{c}5 \\
(14 \cdot 0)\end{array}$ & $\mathbf{0}$ & $\mathbf{0}$ & $\mathbf{0}$ & $\mathbf{0}$ & $\mathbf{0}$ & $\begin{array}{c}4 \\
(14 \cdot 0)\end{array}$ & $\mathbf{0}$ \\
\hline Fusarium oxysporum 1 & $\mathbf{0}$ & $\mathbf{0}$ & $\mathbf{0}$ & $\mathbf{0}$ & $\begin{array}{c}3 \\
(\mathbf{1 2} \cdot 0)\end{array}$ & $\mathbf{0}$ & $\begin{array}{c}5 \\
(15 \cdot 0)\end{array}$ & $\mathbf{0}$ \\
\hline F. oxysporum 2 & $\mathbf{0}$ & $\mathbf{0}$ & $\begin{array}{c}1 \\
(12 \cdot 0)\end{array}$ & $\mathbf{0}$ & $\begin{array}{c}2 \\
(12 \cdot 0)\end{array}$ & $\mathbf{0}$ & $\begin{array}{c}3 \\
(11 \cdot 5)\end{array}$ & $\mathbf{0}$ \\
\hline F. poae & $\mathbf{0}$ & $\begin{array}{c}3 \\
(14 \cdot 0)\end{array}$ & $\mathbf{0}$ & $\mathbf{0}$ & $\mathbf{0}$ & $\mathbf{0}$ & $\begin{array}{c}3 \\
(12 \cdot 0)\end{array}$ & $\mathbf{0}$ \\
\hline Stachybotrys atra & $\mathbf{0}$ & $\begin{array}{c}2 \\
(9 \cdot 5)\end{array}$ & $\mathbf{0}$ & $\mathbf{0}$ & $\stackrel{2}{2}$ & $\mathbf{0}$ & $\begin{array}{c}4 \\
(14 \cdot 0)\end{array}$ & $\mathbf{0}$ \\
\hline $\begin{array}{l}\text { Control (root exudate } \\
\text { only) }\end{array}$ & & & & & & & o & $\begin{array}{c}5 \\
(18 \cdot 0)\end{array}$ \\
\hline
\end{tabular}

Growth of the rhizosphere organisms in a medium consisting of rhizosphere soil extract + root exudate increased their inhibitory capacities (Pl. 1, fig. 4). For example, the stimulation of Fusarium both by an isolate of Stachybotrys atra and one of Fusarium poae, after growth in Czapek-Dox medium, contrasted with the inhibition they caused when the culture medium contained exudate. Gliocladium roseum isolate 2 also caused stimulation when grown in 
soil extract, but not in root exudates. With these fungi, as with all the ten isolates tested, the usual stimulation of race 1 of the pathogenic Fusarium by root exudate from variety Onward was prevented when the root exudate was added to culture filtrates of any of the ten fungi tested. A further feature of this interaction is that the three different strains of Gliocladium roseum, while giving uniform inhibition after culture in media containing root exudate, differed in their reaction towards the Fusarium when grown in liquid cultures containing soil extract. In addition, culture filtrates of some rhizosphere isolates of non-pathogenic $\boldsymbol{F}$. oxysporum inhibited growth of pathogenic Fusarium, but only when the culture media contained pea-root exudates or pea-rhizosphere soil extracts.

\section{DISCUSSION}

Garrett (1936) suggested a quantitative effect of the rhizosphere microflora on the advance of Ophiobolus graminis along wheat roots, and that the ability of any soil organism to produce antagonistic metabolites will depend on the availability of nutrients. He pointed out that any antibiotic metabolites produced by soil microflora are likely to be more concentrated, and therefore of more biological significance, in the immediate vicinity of the organism which produces them (Garrett, 1956). Circumstances conducive to a high rate of metabolism in soil organisms exist in the rhizosphere, and any root pathogen has to contend with the metabolic products of other rhizosphere organisms. Previous results (Buxton, $1957 a, b$ ) suggested that part of the resistance of peas to attack by soil-borne Fusarium oxysporum may act outside the roots by the differential action of root exudates on the Fusarium in the rhizosphere. But under natural conditions root exudates are perhaps unlikely to be the dominant factors in the rhizosphere environment, for the microflora will rapidly change the chemical nature of the exudates and, in doing so, may acquire metabolic activities not normally detected in the laboratory. The results described here show that root exudates from susceptible hosts, which encourage the pathogen's growth or promote germination of its spores, may at the same time increase the inhibitory potentialities of its competitors. But two important factors limit attempts to relate experimental results with what may actually happen in the rhizosphere. First, the current techniques for isolating the rhizosphere microflora are inadequate; they may not reveal all the organisms that proliferate near roots, and the selective nature of any culture medium used to isolate rhizosphere organisms may give an inaccurate picture of the frequency with which each species occurs. Secondly, the concentration either of root exudate or of the inhibitory metabolites from each of the rhizosphere micro-organisms at the root surface cannot be measured. Nevertheless, even with these difficulties, which are inherent in most experiments with soil organisms, the rhizosphere microflora clearly acts differently towards pathogenic Fusarium when under partially simulated rhizosphere conditions than when freed from root exudate or soil extract.

The nearer the root surface, the more likely is Fusarium oxysporum f. pisi to be stimulated by exudates and inhibited by rhizosphere organisms, but 
there may be a threshold value at which the exudates stimulate enough for the Fusarium to withstand the antibiosis. Indeed, if this were not so, infection would be unlikely to occur. In addition, the rhizosphere organisms that are the strongest inhibitors are not necessarily the most prevalent; nor are they likely to be uniformly distributed along the root surface, and 'free' areas may exist in which the pathogenic Fusarium proliferates unhindered and invades the root. Another important consideration is whether the organisms that are the most prevalent at or before the stage when the Fusarium enters the host root are also among the most inhibitory. The relationship between prevalence and antibiotic rating is shown in Fig. 2. Figure $2 \mathrm{~A}$ shows that up to 30 days after germination only a relatively small proportion of the organisms which
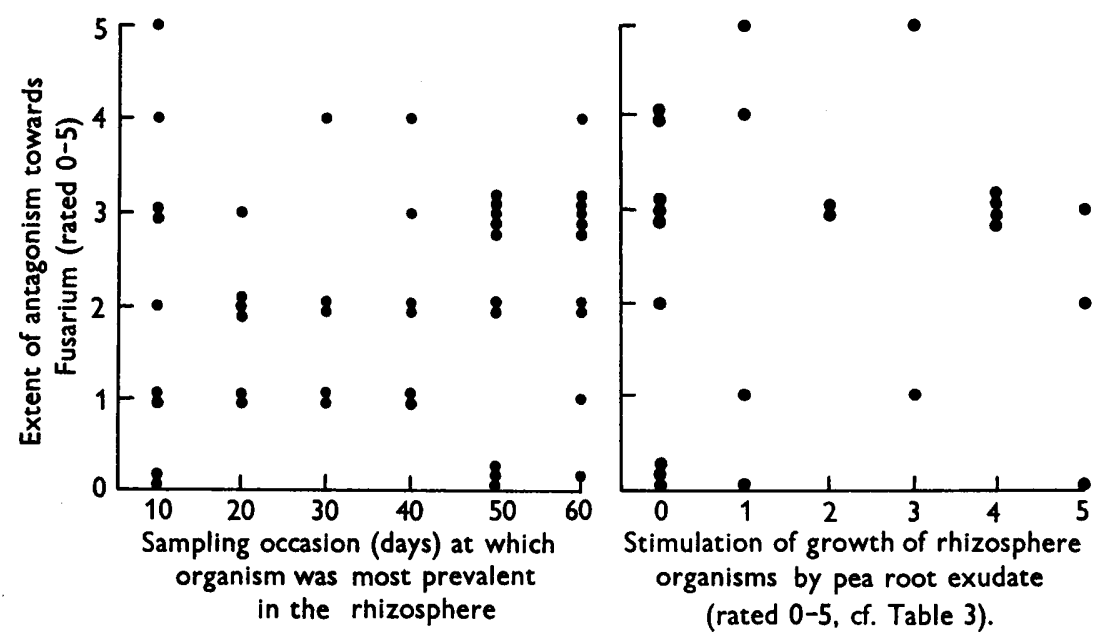

Fig. 2. A. Relation between the prevalence of rhizosphere organisms at different stages of growth of pea variety Onward and their grades of antagonism to Fusarium oxysporum f. pisi race 1. B. Relation between the grade of stimulation of 24 rhizosphere organisms by root exudate from pea variety Onward and their grades of antagonism to F. oxysporum f. pisi race 1.

strongly antagonize Fusarium were prevalent, and although many that are mildly antagonistic occurred early, they were less prevalent than later. After 30 days, by when the Fusarium has entered the root, antagonists are likely to affect the course of infection. Figure $2 \mathrm{~B}$ shows that the extent to which root exudates encouraged the growth of individual micro-organisms was not correlated with the extent to which these organisms antagonized the pathogenic Fusarium. Many of the micro-organisms that were stimulated by root exudate occurred in the earlier samples of rhizosphere soil, which suggests that, although Fusarium may have to contend with antibiotic metabolites from other rhizosphere micro-organisms, an equally important factor controlling its growth in the rhizosphere may be competition for the nutrients in root exudates.

Several isolates of Fusarium oxysporum not only occur in abundance in the rhizosphere, but can inhibit the pathogenic forma specialis of the same species. 
Such an interaction between two very closely related biotypes serves to emphasize the dangers of assuming, as many workers have, that all isolates of $F$. oxysporum from infested soil necessarily belong to the pathogenic strain. Many earlier surveys of soil made to assess the prevalence of pathogenic $F$. oxysporum have little value because of failure to recognize that morphological similarity is no criterion of physiological identity. Not only do many isolates of any single species of rhizosphere fungus inhibit the pathogenic Fusarium, but different strains within the one species differ in their ability to inhibit, a phenomenon that was especially evident among isolates of $F$. oxysporum and Gliocladium roseum. In addition to possessing physiologic races which differ in pathogenicity, $F$. oxysporum seems also able to contain strains that differ in their abilities to colonize the rhizosphere.

I thank Miss Alison M. Fletcher for technical assistance and Mr V. Stansfield for taking the photographs.

\section{REFERENCES}

Audus, L. J. (1946). A new soil perfusion apparatus. Nature, Lond. 158, 419.

Buxton, E. W. (1957a). Some effects of pea root exudates on physiological races of Fusarium oxysporum Fr. f. pisi (Linf.) Snyder \& Hansen. Trans. Brit. mycol. Soc. 40, 145.

Buxton, E. W. (1957b). Differential rhizosphere effects of three pea cultivars on physiologic races of Fusarium oxysporum f. pisi. Trans. Brit. mycol. Soc. 40, 305.

Garretr, S. D. (1936). Soil conditions and the take-all disease of wheat. Ann. appl. Biol. 23, 667.

GarretT, S. D. (1956). Biology of Root-Infecting Fungi. Cambridge University Press.

Romвours, J. E. (1953). The microorganisms in the rhizosphere of banana plants in relation to susceptibility or resistance to Panama disease. Plant \& Soil, 4, 276.

Rovira, A. D. (1956). Plant root excretions in relation to the rhizosphere effect. II. A study of the properties of root exudate and its effect on the growth of micro-organisms isolated from the rhizosphere and control soil. Plant \& Soil, $7,195$.

Stenton, H. (1958). Colonization of roots of Pisum sativum L. by fungi. Trans. Brit. mycol. Soc. 41, 74 .

Timonin, M. I. (1941). The interaction of higher plants and soil microorganisms. III. Effects of by-products of plant growth on activity of fungi and actinomycetes. Soil. Sci. 52, 395.

WARCUP, J. H. (1950). The soil-plate method for isolation of fungi from soil. Nature, Lond. 166, 117.

\section{EXPLANATION OF PLATE 1}

Fig. 1. A. Fusarium oxysporum f. pisi race 1 inhibited by root exudate from pea variety Wilt-Resistant Alaska, which it cannot infect. Right-hand cylinder contains exudate heated for $10 \mathrm{~min}$. at $100^{\circ}$. B. F. oxysporum f. pisi race 2, showing increased growth near cylinders, which contain root exudate from pea variety Wilt-Resistant Alaska, which it infects.

Fig. 2. Soil perfusion apparatus (as described by Audus, 1946) containing peas variety Onward growing in sterile washed sand $\left(x \frac{1}{6}\right)$. Vacuum pump connexion to $X$ results in water movement through tube on left. Water drips over roots from $Y$ and root exudate collects in $\mathbf{Z}$. 
Journal of General Microbiology, Vol. 22, No. 3

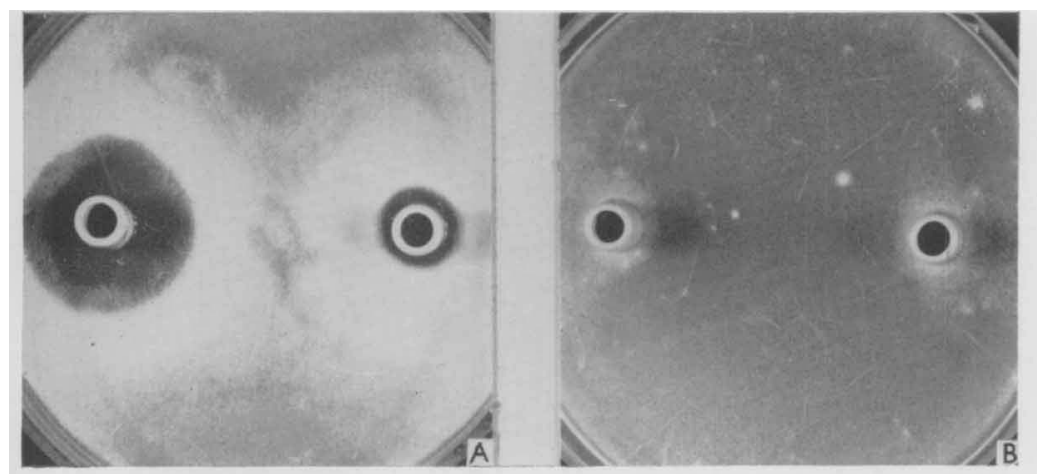

Fig. 1
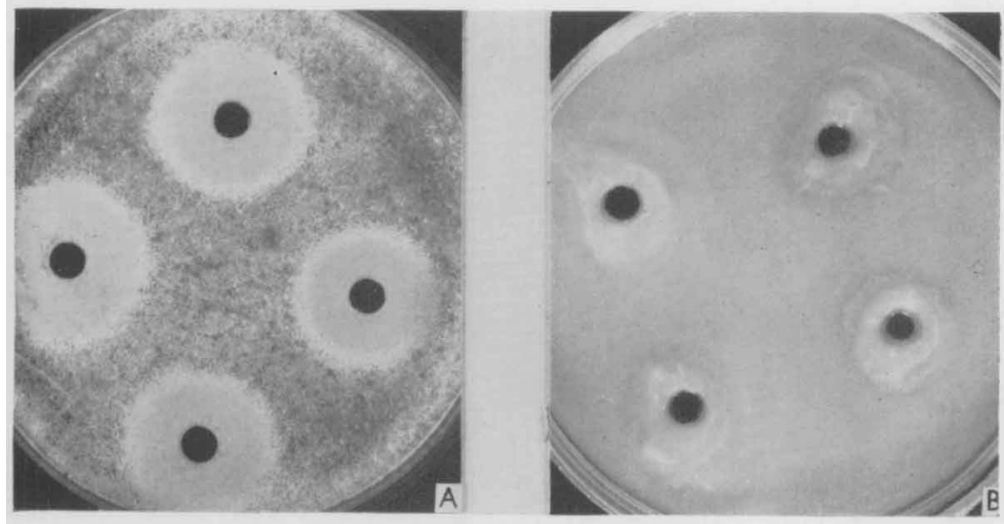

Fig. 3

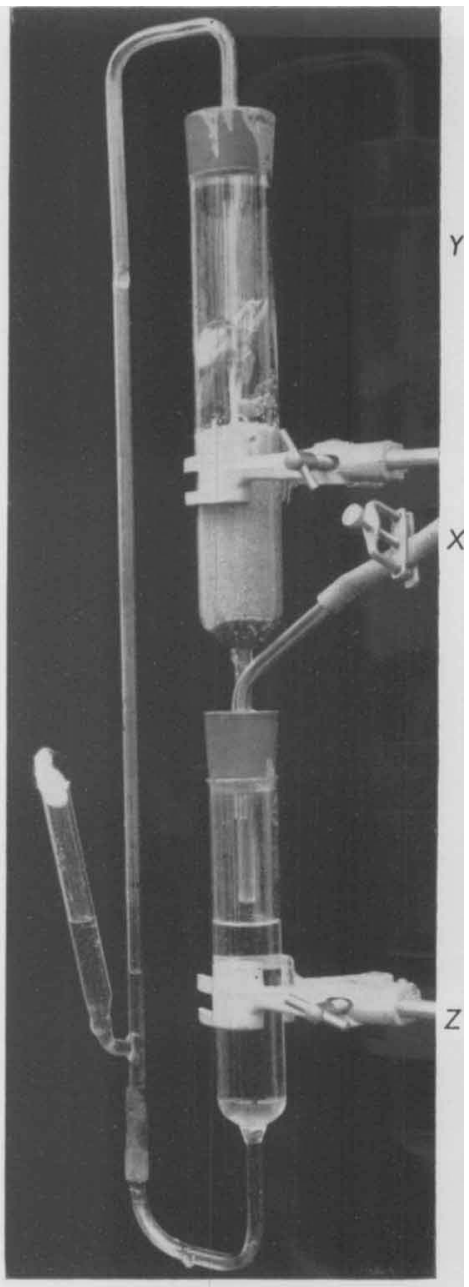

Fig. 2
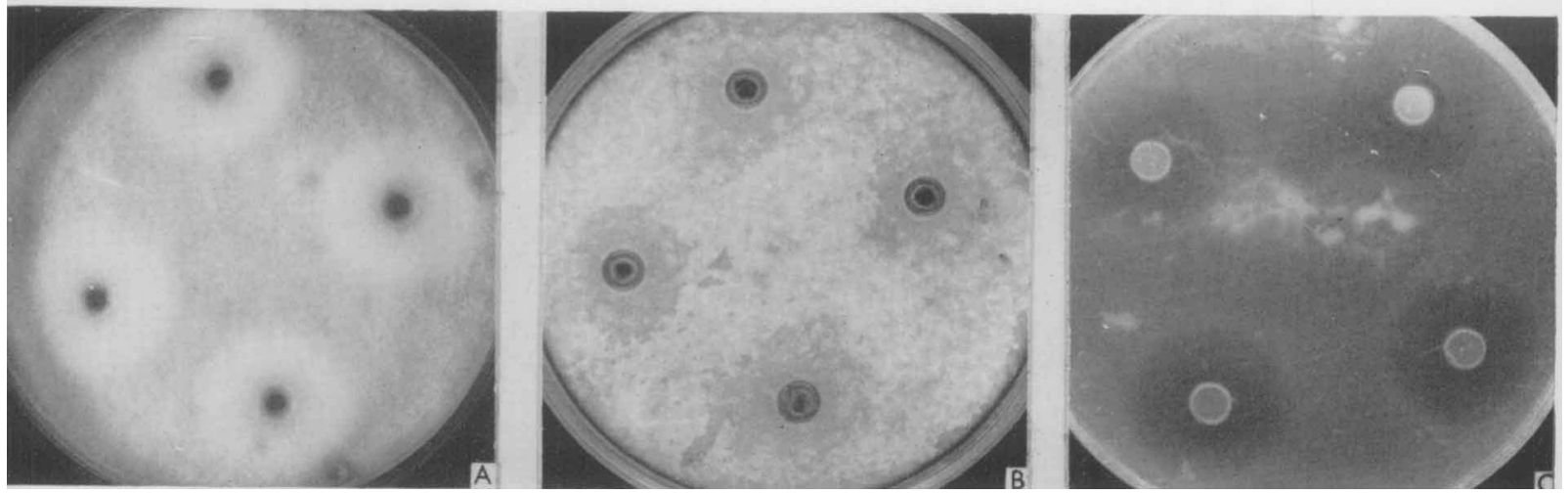

Fig. 4.

E. W. Buxton-Fusarium oxysporum in rimizosphere of pea. Plate 1 
Fig. 3. Stimulatory effect of root exudate from pea variety Onward on an unidentified pea rhizosphere organism (A) and on a rhizosphere bacterium (B). Both organisms were dispersed through the agar when poured, and exudate was added $2 \mathrm{hr}$. later to the holes.

Fig. 4. A. Stimulatory effect of root exudate from pea variety Onward on $F$. oxysporum f. pisi. B. Inhibitory effect on F. oxysporum f. pisi of Gliocladium roseum culture filtrate when grown in soil extract. C. Increased inhibition by culture filtrate from the same organism when grown in soil extract + pea-root exudate.

(Received 27 October 1959) 